\title{
Les invariants et les covariants, en qualité de critères pour les racines d'une équation
}

(par H. Schramm, prof. i Wiener-Neustadl).

(Continuazione.)

III.

Pour compléter la discussion des équations algébriques par moyen de critères invariantifs (*), il nous reste encore à trouver une espèce d'invariants, indiquant la coëxistence de $r$ groupes à $s$ racines égales.

Posons d'abord, pour faciliter la déduction, $r=3, s=4$, et admettons qu'il y a un invariant doué de la propriété d'avoir la valeur zéro, lorsque l'équation, à la forme de laquelle il appartient, contient 3 groupes à 4 racines égales; cet invariant aura à conserver une valeur sensible pour

$$
\begin{aligned}
& 1=2=3=4 \\
& 5=6=7=8 \\
& 9=10=11, \quad \text { et } \quad 11>12
\end{aligned}
$$

en denotant les racines $\alpha_{1}, \alpha_{2}, \alpha_{3}, \ldots$ de l'équation, simplement par $1,2,3, \ldots$

Posons de plus, qu'on ait représenté l'invariant cherché en fonction des differences des racines $\alpha_{1}, \alpha_{2} \ldots$, savoir :

$$
I^{(r, s)}=\mathbf{\Sigma} f\left(\alpha_{1}-\alpha_{2}\right)\left(\alpha_{1}-\alpha_{3}\right) \ldots ;
$$

il est évident, que sous la dite condition la plus grande partie des termes de la somme $\Sigma$ se réduira à zéro, et qu'il n'en restent exceptés que les termes,

(*) Annali di Matematica pura ed applicata: Serie II.", t. 1.", pag. 250 a pag. 279. Annali di Matematica, tomo III. 
daus lesquels manqueront toutes les différences formées des racines: $1,2,3,4$; de plus, les différences formées des racines $5,6,7,8$, et encore les différences formées des racines $9,10,11$.

Ces termes-ci ne contiendront donc que les différences suivantes:

(a.) Les différences formées des $(r s-s)$ racines: $1,2,3,4$, et $5,6,7,8$, en combinant toujours une racine du groupe $1,2,3,4$ avec une des $5,6,7,8$; cela donne en tout

$$
\frac{(r s-s)(r s-s-1)}{1 \cdot 2}-(r-1) \cdot \frac{s(s-1)}{2}=\frac{s^{2}}{2}(r-1)(r-2) \quad \text { différences. }
$$

(b.) Les différences formées des $(r s-s)$ racines: $1,2,3, \ldots 7,8$, avec les $(s-1)$ racines: $9,10,11$;

en tout $(s-1)(r s-s)=s(s-1)(r-1)$ différences.

(c.) Les $s(r-1)(n-r s+1)$ différences formées des $(r s-s)$ racines: $1,2, \ldots 7,8$, avec les $(n-r s+1)$ racines: $12,13, \ldots n$.

(d.) Les $(s-1)(n-r s+1)$ différences formées des $(s-1)$ racines: $9,10,11$, avec les $(n-r s+1)$ racines: $12,13, \ldots n$.

(e.) Les $\frac{1}{2}(n-r s)(n-r s+1)$ différences, composées des $(n-r s+1)$ racines: $12,13, \ldots n$.

En dénotant encore, pour abréger, les produits des différences développées en $(a),(b),(c) \ldots$ par

$$
\begin{aligned}
& A=(1,5)(1,6) \ldots(r s-2 s, r s-s) \\
& B=(1,9)(1,10) \ldots(r s-s, r s-1) \\
& C=(1,12)(1,13) \ldots(r s-s, \quad n) \\
& D=(9,12)(9,13) \ldots(r s-1, \quad n) \\
& E=(12,13)(12,14) \ldots(n-1, \quad n)
\end{aligned}
$$

l'invariant en question prendra la forme:

$$
I^{(r, s)}=\sum A^{u} B^{v} C^{x} D^{y} E^{z} .
$$

Mais il faut de plus, que chaque racine entre $p$ fois dans chaque terme de la somme $\Sigma ; p$ dénotant le degré de l'invariant par rapport aux coefficients de l'équation. 
Schramm: Gritères pour les racines d'une équation.

En faisant donc des $n$ racines de l'équation les trois parties:

$$
\begin{aligned}
& \text { (I) } \ldots \ldots \ldots \ldots \quad 1, \quad 2, \quad 3, \quad \ldots \ldots \ldots(r s-s) \\
& \text { (II) } \ldots \ldots \ldots(r s-s+1),(r s-s+2) \ldots \ldots(r s-1) \\
& \text { (III) } \ldots \ldots \ldots n r s, \quad r s+1, \ldots \ldots \ldots n
\end{aligned}
$$

il est facile à voir, qu'une quelconque des racines de la partie (I) entrera dans le produit $A, \ldots \ldots s(r-2)$ fois

$$
\begin{aligned}
& \text { » } B, \quad s-1 \text { » } \\
& \text { » } \quad \text {, } m-r s+1 \text { » }
\end{aligned}
$$

De même une racine de la partie (II) entrera

$$
\begin{aligned}
& \text { en } B \ldots \ldots s(r-1) \text { fois } \\
& \text { » } D \ldots \ldots n-r s+1 \text { 》 }
\end{aligned}
$$

et une racine de la partie (III) entrera

$$
\begin{aligned}
& \text { en } C \ldots \ldots s(r-1) \text { fois } \\
& \gg D \ldots \ldots s-1\rangle \\
& \gg E \ldots \ldots n-r s 》
\end{aligned}
$$

Il en résulte, que les exposants $u, v, x, \ldots$ sont liés aux conditions suivantes:

$$
\begin{aligned}
s(r-2) u+(s-1) v+(n-r s+1) x & =p \\
s(r-1) v+(n-r s+1) y & =p \\
s(r-1) x+(s-1) y+(n-r s) \quad z & =p .
\end{aligned}
$$

La première de ces équations $(p)$ représente la somme des exposants d'une racine de la partie (I), la seconde et la troisième la somme des exposants d'un racine resp. en (II) et en (III).

Les invariants déterminés par ces équations auront la propriété de prendre la valeur zéro lorsqu'il y a dans l'équation $r$ suites à s racines égales, et ils conserveront une valeur sensible lorsque les $r$ suites de racines ne sont pas completes. Aussi il va sans dire qu'ils restent égales à zéro pour des valeurs plus grandes que $r$ et $s$.

On peut vérifier la correspondance de ces équations générales avec celles 
trouvées dans l'article précédent, en posant $s=2$, et $r+1$ au lieu de $r$. La seconde et la troisième équation représentera alors identiquement la même condition, en posant encore $x=v \cdot y=z$, et il n'en restent que les deux équations seules:

$$
\begin{aligned}
& 2(r-1) u+(n-2 r) v=p \\
& 2 r v+(n-2 r-1) z=p .
\end{aligned}
$$

Ce sont on verité les mêmes équations, moyennant desquelles nous avions calculé les invariants designés par $I^{(r)}$ (II, pag. 272).

Pour appliquer ces invariants dans la discussion des équations algébriques il sera utile de calculer oncore la somme des exposants dans les produits développés de chaque terme de l'invariant. En dénotant par $S$ cette somme des exposants, on aura

$$
\begin{gathered}
S=\frac{1}{2} s^{2}(r-1)(r-2) u+s(s-1)(r-1) v+s(r-1)\left(n-r^{2} s+1\right) x+(s-1)(n-r s+1) y \\
+\frac{1}{2}(n-r s+1)(n-r s) z .
\end{gathered}
$$

Mais on peut transformer cette expression de la maniere suivante:

$$
\begin{aligned}
& S=\frac{1}{2} s^{2}(r-1)(r-2) u+\frac{1}{2} s(r-1)(s-1) v+\frac{1}{2} s(r-1)(n-r s+1) x+ \\
& +\frac{1}{2} s(s-1)(r-1) v+\frac{1}{2}(s-1)(n-r s+1) y+ \\
& \quad+\frac{1}{2} s(r-1)(n-r s+1) x+\frac{1}{2}(s-1)(n-r s+1) y+\frac{1}{2}(n-r s+1)(n-r s) z \\
& S=\frac{1}{2} s(r-1) p+\frac{1}{2}(s-1) p+\frac{1}{2}(n-r s+1) p \\
& \quad S=\frac{n p}{2} .
\end{aligned}
$$

Cette équation est valable comme on sait pour chaque autre espece d'invariants; car il est évident, qu'un terme quelconque en $\Sigma$ contient chacune des $n$ racines $p$ fois, ce qui fait, en tout, $n p$ racinos; mais comme celles-ci sont combinées par deux dans une différence, elles donnent un produit de

$$
S=\frac{n p}{2}
$$

facteurs linéaires. Dans un invariant, qui doit conscrver son signe, même quand on change les signes de toutes les racines de l'équation, il faut que $S$ soit un nombre pair, et le degré $p$ d'un tel invariant sera lié à la condition:

$$
p=\frac{4 s_{i}}{n}
$$


c'est à dire :

$$
\begin{aligned}
& p=4 p_{1}, \quad \text { pour } n=2 n+1 \\
& p=2 p_{1} \quad » \quad n=4 m+2 \\
& p=p_{1} \quad \gg n=4 m
\end{aligned}
$$

$p_{1}$ dénotant un nombre entier quelconque. C'est aussi la condition sous laquelle un invariant sera applicable dans la discussion des équations, parceque toute autre invariant aura identiquement la valeur zéro, ou pour des racines quelconques $\alpha_{1}, \alpha_{2}, \alpha_{3}, \ldots$ ou au moins pour la supposition :

$$
\begin{aligned}
& \alpha_{1}=-\alpha_{2} \\
& \alpha_{3}=-\alpha_{4} \\
& \ldots . \\
& \alpha_{n-1}=-\alpha_{n}\left(^{*}\right)
\end{aligned}
$$

Les formules générales pour le degré $p$ deviennent impraticables pour $r=1$, ou mieux dit, elles prennent une forme plus simple; car, dans ce cas particulier, il n'y aura plus de racines dans la partie (I) et par conséquent les trois équations $(p)$ se réduiront aux deux seules:

$$
\begin{aligned}
(n-s+1) y & =p \\
(s-1) y+(n-s) z & =p .
\end{aligned}
$$

Cette espèce d'invariants aura la forme

$$
\begin{aligned}
& I^{(1, s)}=\mathbf{\Sigma} D^{y} E^{z} \\
& D=(1, s)(1, s+1) \ldots(s-1, n) \\
& E=(s, s+1)(s, s+2) \ldots(n-1, n)
\end{aligned}
$$

avec les valeurs des exposants $y$ et $z$ :

$$
y=\frac{p}{n-s+1}, \quad z=\frac{p(n-2 s+2)}{(n-s)(n-s+1)} .
$$

(*) On trouve dans les Flemente der neueren Gcometrie, etc., von D. ${ }^{\circ}$ W. FredLER, p. 160, un invariant du degré $p=3$ appartenant à une forme du $6^{\text {me }}$ degré:

$$
I_{6,3}=a_{0} a_{2} a_{6}-3 a_{0} a_{3} a_{5}+2 a_{0} a_{4}^{2}-a_{4}^{2} a_{6}+3 a_{1} a_{2} a_{5}-a_{1} a_{3} a_{4}+2 a_{2} a_{3}^{2}-3 a_{9}^{2} a_{4}
$$

Mais ce n'est qu'un semi-invariant résultant de la transformation linéaire $\alpha=\alpha x+\beta$ et $y$ constant. On réconnait sur le champ son variabilite, en changeant les coeffeients $a_{0}$, $w_{1}, a_{3} \ldots$ en $a_{6}, a_{5}, a_{4} \ldots$ 
46 Schramm: Critères pour les racines d'une équation.

On prendra pour $p$ un nombre entier, qui donne aussi aux exposants $y$ et $z$ une valeur positive et entière. Cela ne sera possible que pour

$$
2 s<n+2
$$

parceque pour $2 s>n+2, z$ devient negatif.

Voilà une solution du probléme proposé; mais on ne peut pas dire qu' elle soit la solution la plus générale, parcequ'il y a pour la déduction des invariants de la dite propriété plusieurs voies, qui mènent à des résultats en quelque part différents.

Nous en donnerons un exemple pour le cas $r=1$, et $s$ quelconque. Posons à ce but, qu'on ait fait des $n$ racines de l'équation $(s-1)$ parties dont les premières $(s-2)$ parties contiennent $\rho$ racines chacune, et la dernière le reste:

$$
\begin{array}{llc}
1, & 2, & 3, \ldots \rho \\
\rho+1, & \rho+2, \ldots \ldots & 2 \rho \\
\ldots \ldots \ldots & \ldots \ldots \\
(s-2) \rho+1, & (s-2) \rho+2, \ldots(s-1) \rho, \ldots n-1, \quad n .
\end{array}
$$

En formant le produit de toutes les différences de chaque partie, on obtient:

$$
\begin{aligned}
& A_{1}=(1,2)(1,3) \ldots(\rho-1, \rho) \\
& A_{2}=(\rho+1, \rho+2)(\rho+1, \rho+3) \ldots(2 \rho-1,2 \rho) \\
& \ldots \ldots \ldots \ldots \ldots \ldots \ldots \ldots \ldots \ldots \ldots \\
& A_{s-1}=[(s-2) \rho+1,(s-2) \rho+2] \ldots \ldots[n-1, n]
\end{aligned}
$$

Ces produits donneront un invariant de la forme

$$
I_{p}^{(\varrho)}=\sum A_{1}^{x} A_{2}^{x} \ldots A_{s-2}^{x} A_{z-1}^{y}
$$

en supposant les exposants $x$ et $y$ soumis à la condition :

$$
(\rho-1) x=[n-(s-2) \rho-1] y=p \text {. }
$$

Il y a une différence essentielle entre ces invariants, et ceux trouvés par la déduction précédente; et pourtant ils ont la propriété commune d'avoir la valeur zéro pour $s$ racines égales. Car en supposant parmi les $n$ racines d'une équation $s$ racines égales, il est évident que l'un ou l'autre des produits $A_{1}, A_{z}, A_{s-1}$ contiendra deux ou plus racines égales et par conséquent chaque terme de la somme $\mathbf{\Sigma}$ aura zéro en facteur. 
Mais en examinant la dernière équation, on roit que la valeur de $\rho$ peut varier entre les limites:

$$
\rho>1, \quad \rho<\frac{n-1}{s-2}
$$

et qu'il y aura dans certains cas plusieurs invariants différents, pour la même valeur des. En posant pour $\rho$ la plus simple valeur $\rho=2$, on aura pour $x$ et $y$ les équations:

$$
x=p, \quad y=\frac{p}{n-2 s+3} .
$$

Ces invariants auront donc en général le degré

$$
p=(n-2 s+1) p_{1}
$$

pendant que les autres, nommés $I^{(1, s)}$, seront du degré $p^{\prime}=(n-s)(n-s+1) p_{1}$, ce qui donne en général $p<p^{\prime}$.

IV.

Passons maintenant à la récherche des critères invariantifs, indiquants la coëxistence de plusieurs racines égales et imaginaires.

Nous avions exposé dans l'art. II de ce travail, que les invariants $I^{(r)}$ indiquent par le changement de signe l'existence de $2(r+1)$ racines imaginaires dans l'équation donnée, et qu'ils s'évanouissent lorsque les $2(r+1)$ racines sont deux à deux égales. Mais dans ce dernier cas, ils perdent aussi leur faculté de servir comme indices pour le nombre des racines imaginaires, et il faut qu'on les remplace par une autre espéce d'invariants.

Il est pourtant très difficile à donner des formules générales qui renferméraient tous les cas possibles, pour chaque combinaison de racines égales, et en partie imaginaires, et nous ne chercherons par conséquent que les indices dans le cas spécial, quand l'équation donnée contient $r$ parties a 4 racines imaginaires et deux à deux égales.

En supposant donc dans cette équation $r$ groupes à quatre racines imaginaires deux à deux égales, on sait que celles-ci auront la forme:

$$
\begin{aligned}
& \alpha_{1}=\alpha_{3}=p+q \sqrt{-1} \\
& \alpha_{2}=\alpha_{1}=p-q \sqrt{-1}
\end{aligned}
$$


et en faisant $q=0, q_{1}=0, \ldots$ ces racines seront réelles et $q u$ atre à $q u a t r e$ égalos:

$$
\alpha_{1}=\alpha_{2}=\alpha_{3}=\alpha_{4} .
$$

Il faut donc que l'invariant cherche passe par zéro quand il y a 4 groupes à 4 racines égales dans l'équation, et il faut qu'il change le signe au moment, où l'on donne à $q, q_{1}, q_{2}, \ldots$ une valeur sensible.

Ces invariants résultéront des équations générales $(p)$, en y posant $s=4$ :

$$
\begin{aligned}
4(r-2) u+3 v+(n-4 r+1) x & =p \\
4(r-1) \quad v+(n-4 r+1) y & =p \\
4(r-1) x+3 y+(n-4 r) \quad z & =p .
\end{aligned}
$$

On déduira aussi la valeur de l'exposant $\mu$ dans la plus haute puissance du produit développé des quantités imaginaires :

$$
q q_{1} q_{2} \ldots(\sqrt{-1})^{u}
$$

en ayant ígard à ce que nous avons expliqué dans l'art. II de ce travail, pag. 273, savoir que l'on trouve cette puissance dans un terme de la somme ¿qui contient le plus petit nombre de différences formées de deux racines imaginaires. Cet exposant plus haut aura en général les valeurs suivantes:

1. En supposant $4 r-2$ racines imaginaires dans l'équation donnée:

$$
u_{1}=8(r-1)(r-2) u+12(r-1) v+4(r-1)(n-4 r+1) x+(n-4 r+1) y .
$$

2. En supposant $4 r+2 \sigma$ racines imaginaires dans léquation:

$$
\begin{gathered}
\mu_{2}=8(r-1)(r-2) u+12(r-1) v+4(r-1)(n-4 r+1) x+3(n-4 r+1) y \\
+(2 \sigma+1)(n-4 r-\sigma) z
\end{gathered}
$$

ou aussi plus en abrégé, en plaçant $4 t$ au lieu des expressions avec le coefficient 4 :

$$
\begin{aligned}
& \mu_{1}=4 t+(n-4 r+1) y \\
& \mu_{2}=4 t+3(n-4 r+1) y+(2 \sigma+1)(n-4 r-\sigma) z .
\end{aligned}
$$

Chaque invariant de cette espèce doit rester positif pour $4 r-2$ racines imaginaires, mème en supposant les quantités $q, q_{1}, q_{2}, \ldots$ infiniment grandes; il faut donc que $\mu_{1}$ soit un nombre de la forme

$$
4 t, \text { ou } 4 t+1 \text {. }
$$


Car en supposant $\mu_{1}=4 t$, l'invariant $I^{(r, 4)}$ aura pour

$$
\begin{array}{ll}
\alpha_{1}=\alpha_{3}=q \sqrt{-1}, & \alpha_{5}=\alpha_{7}=q_{1} \sqrt{-1} \\
\alpha_{2}=\alpha_{4}=-q \sqrt{-1}, & \alpha_{6}=\alpha_{8}=-q_{1} V-1
\end{array}
$$

le même signe qu'il a dans le cas où l'on suppose :

$$
\begin{aligned}
& \alpha_{1}=\alpha_{3}=q, \quad \alpha_{5}=\alpha_{7}=q_{1} \\
& \alpha_{2}=\alpha_{4}=-q, \quad \alpha_{6}=\alpha_{8}=-q_{1} \\
& \ldots \ldots \ldots \ldots
\end{aligned}
$$

(toujours pour des valeurs très grandes de $q, q_{1}, \ldots$ ), ou, pour l'exprimer plus en général, il aura pour $4 r-2$ racines imaginaires le mème signe que pour $n$ racines réelles.

La seconde supposition $\mu_{1}=4 t+1$ donne au produit $q q_{1} \ldots(\sqrt{-1})^{\mu}$ une valeur imaginaire; mais on sait qu'un invariant reste réel pour de valeurs réelles des coefficients de l'équation. Il en suit, que les puissances impaires de $\sqrt{-1}$ s'évanouissent, et que le signe de l'invariant dépendra de la puissance $(\sqrt{-1})^{\mu-1}$, ce qui conduit au même cas : $\mu-1=4 t$.

En consequence dans le choix des valeurs de $y$ il faut avoir égard anx conditions :

$$
(n-4 r+1) y=4 t, \text { ou }(n-4 r+1) y-1=4 t .
$$

Le même invariant doit changer le signe pour $4 r+2 \sigma$ racines imaginaires, et afin que cela soit possible, il faut que $\mu_{2}$ ait la forme:

$$
\mu_{2}=4 t+2 \text { ou } \mu_{2}-1=4 t+2 \text {. }
$$

Considérons la première de ces conditions, en remettant la seconde à la discussion des équations de degré particulier, et posons $\mu_{1}=4 t, \mu_{2}=4 t+2$. Ces équations seront satisfaites en faisant:

$$
(n-4 r+1) y=4 t \text {, et }(n-4 r-\sigma) z=4 t+2 .
$$

Pour $n=2 m+1$, et $\sigma=2 \sigma_{1}$, on aura à satisfaire ontre que les équations $(p)$, encore les conditions:

$$
\begin{gathered}
(2 m-4 r+2) y=4 t \\
(2 m-4 r-2 \sigma+1) z=4 t+2
\end{gathered}
$$


ce que l'on peut toujours faire en posant $z=4 z_{1}+2$, et il sera alors:

$$
\begin{aligned}
& \text { pour } 4 r+4 \sigma_{1} \text { racines imaginaires, } \mu_{2}=4 t+2 \\
& \text { pour } 4 r+4 \sigma+2 》 \quad \gg \quad \mu_{2}=4 t \text {. }
\end{aligned}
$$

Tels invariants auront la faculté d'indiquer par un changement de signe la coëxistence de $4 r$ racines imaginaires, et lorsqu'il est en même temps $I^{\left(r_{1}\right)}=0$, posant $2 r-1=r_{1}$, ces $4 r$ racines seront deux à deux égales.

Les équations du degré $n=2 m$ donnent les conditions:

$$
\begin{gathered}
(2 m-4 r+1) y=4 t, \quad \text { ou } \quad y=4 y_{1} \\
\text { et } \mu_{2}=4 t+3(2 m-4 r+1) y+\left(2 \sigma_{1}+1\right)(2 m-4 r-\sigma) z=4 t+2 .
\end{gathered}
$$

Mais en posant $\sigma=0$, ce qui correspond à $4 r$ racines imaginaires, on déduira la valeur de $(2 m-4 r) z$ des deux dernières équations pour le degré $(p)$, savoir :

$$
(2 m-4 r) z=4(r-1)(v-x)+(2 m-4 r-2) 4 y_{1}=4 t
$$

et il sera pour $y=4 y$, toujours $\mu_{2}=4 t$.

Quant au second cas, lorsqu'il est $\mu_{2}$ impair, on aura à satisfaire la condition :

$$
(2 m-4 r+1) y-1=4 t
$$

ce qui donne :

$$
\mu_{2}=4 t+1+2(m-2 r) z \text {. }
$$

Cette fois il sera bien possible de donner à $\mu_{2}$ la forme $4 t+3$, en supposant $m$ et $z$ impair; et puisque cette puissance impaire de $V=1$ disparait, il est évident que le signe de l'invariant dépendra de la puissance inférieure avec l'exposant:

$$
\mu_{2}-1=4 t+2 .
$$

Mais pour $m$ pair, savoir pour les dégrés $n=4 m_{1}$ on trouve la valeur de $\mu_{2}$ :

$$
\mu_{\mathrm{a}}=4 t+1+4\left(m_{1}-r\right) z=4 t^{\prime}+1
$$

C'est ce qui nous apprend que les invariants $I^{(r, 4)}$, lorsqu'ils appartiennent aux équations du dégré $4 \mathrm{~m}$, conservent pour $4 \mathrm{r}$ racines imaginaires le mème signe que pour $4 m$ racines reelles, en sup- 
posant $q, q_{1}, \ldots$ infiniment grands. Ces invariants ne seront plus applicables en qualité d'indices des racines imaginaires.

On fera de même usage des formules données en III., quand il s'agit de trouver des critères invariantifs pour $6 r, 8 r, 10 r, \ldots$ racines imaginaires, trois à trois, quatre à quatre,... égales, en posant dans les équations $(p)$ respectivement $s=6, s=8, s=10, \ldots$

Dans le cas particulier: $r=1$, on préférera la seconde espèce d'invariants dénotée par $I^{(\rho)}$ et déterminée, en posant $s=3$, par les équations

$$
\begin{gathered}
(\rho-1) x=(n-\rho-1) y=p \\
I_{p}^{(\varrho)}=\mathbf{\Sigma} A_{1}^{x} A_{2}^{y} \\
\mu=(2 \rho-3) x+(2 n-2 \rho-3) y
\end{gathered}
$$

Par rapport à l'équation $(\rho-1) x=(n-\rho-1) y$, la valeur $\bullet$ de $\mu$ sera transformée en

$$
\mu=4(\rho-1) x-(x+y) .
$$

Afin que l'invariant $I^{(}\left({ }^{\prime}\right)$ puisse changer le signe pour 4 racines imaginaires, il faut qu'on ait:

$$
x+y=4 t+2, \text { ou } \quad x+y-1=4 t+2 .
$$

V.

Application des formules précédentes, à la discussion de quelques équations du dégré impair.

1. Soit $n=5$. Les équations du $5^{\text {me }}$ dégré n'ont d'autres invariants de la forme $I^{(r, s)}$, que celui pour $r=1$, et $s=3$, savoir :

$$
I_{12}^{(r, s)}=\Sigma D^{4} G^{2}
$$

Mais c'est identiquement le même invariant du degré $p=12$, que nous avions designé par $I_{12}^{\prime}$ dans l'art. II. de ce travail.

Les autres équations déterminant les invariants dénotés par $I^{(\varrho)}$ donnent pour :

$$
\rho=2, \quad s=3, \quad I_{4}^{(\rho)}=\mathbf{\Sigma} A_{1}^{4} A_{2}^{2}
$$


et les valeurs des produits $A_{1}$ et $A_{2}$ résulteront des formules générales :

$$
A_{1}=(1,2), \quad A_{2}=(3,4)(4,5)(3,5) .
$$

Ici on voit, miex peut-être que des formules précédents, qu'il y a déjà dans les équations du $5^{\text {me }}$ dégré, pour les cas de trois racines égales, deux criteres differents et irréductibles, savoir :

$$
I_{12}^{\prime}=0 \text {, et } I_{4}^{(\rho)}=0 .
$$

Le dernier invariant sera encore un critère pour 4 racines imaginaires car on trouve des équations développées en IV., en posant $\rho=2$

$$
\begin{gathered}
x=[5-3] y=4 p_{1} \\
\mu=4 x+(x+y)=4 t+2 .
\end{gathered}
$$

Ces conditions seront satisfaites en prenant $p_{1}=1, x=4, y=2$, et l'invariant dénoté par le symbole:

$$
I_{4}^{(\rho)}=\mathbf{\Sigma} A_{1}^{4} A_{2}^{2}
$$

indiquera par un changement de signe l'existence de 4 racines imaginaires dans les équations du $5^{\text {me }}$ dégré, et lorsqu'il est en mème temps $I_{12}^{\prime}=0$ ces racines seront deux à deux égales.

Voila donc encore le troisième critère invariantif de M.r SrLvester, résultant de nos équations générales.

Les équations du dégré $n=7$ ont les invariants suivants de la forme $I_{p}^{(r, 8)}:$

$$
\begin{array}{lll}
\text { pour } r=1, & s=3, & I_{20}^{(r, s)}=\Sigma D^{4} E^{3} \\
\text { pour } r=1, & s=4, & I_{12}^{(r, s)}=\Sigma D^{3} E \\
\text { pour } r=2, & s=3, & I_{20}^{(r, s)}=\Sigma B^{6} C^{4} D E^{6} .
\end{array}
$$

Les mèmes équations auront encore les invariants dénotés par le symbole $I_{p}^{(\rho)}$ :

$$
\begin{array}{rlll}
\text { pour } \rho=2 & s=3 & I_{4}^{(\rho)}=\Sigma A_{1}^{4} A_{2} \\
» & \rho=3 & s=3 & I_{12}^{(\rho)}=\Sigma A_{1}^{6} A_{2}^{4} \\
» & \rho=2 & s=4 & I_{4}^{(\rho)}=\sum A_{1}^{4} A_{2}^{4} A_{2}^{2} .
\end{array}
$$


On aura donc les critères invariantifs des équations du 'qme degré indiquants plusieurs racines égrales :

Lorsqu'il est: $I_{20}^{(r, s)}=\mathbf{\Sigma} D^{4} E^{3}=0$

ou aussi: $\quad I_{4}^{(\varrho)}=\mathbf{\Sigma} A_{1}^{4} A_{2}=0$ l'équation contient trois racines égales

$» \quad I_{12}^{(\rho)}=\mathbf{\Sigma} A_{1}^{6} A_{2}^{4}=0$

et pour : $\quad I_{12}^{(r, s)}=\mathbf{\Sigma} D^{3} E=0$

ou aussi: $\left.\quad I_{4}^{(\rho)}=\mathbf{\Sigma} A_{1}^{4} A_{2}^{4} A_{3}^{2}=0\right\} \quad \gg \quad$ quatre racines égales

et encore pour $I_{20}^{(r, s)}=\mathbf{\Sigma} B^{6} C^{4} D E^{6}=0$, l'équation aura deux groupes à trois racines égales.

Il est remarquable que tous ces invariants sont irreductibles, bien qu'ils possèdent quelques propriétés communes. Car en considérant p. e. les trois premièrs invariants, on voit que $I_{20}^{(r, s)}=\mathbf{\Sigma} D^{4} E^{3}$ s'evanouit déjà en y supposant deux paires à deux racines égales, pendant que l'invariant

$$
I_{4}^{(\rho)}=\sum(1,2)^{4} \times(3,4)(3,5) \ldots(6,7)
$$

conserve une valeur sensible. Mais celui-ci devient égale à zéro pour trois paires de racines égales savoir en posant p. e.

$$
1=4, \quad 2=5, \quad 3=6
$$

parceque alors chaque terme contiendra au moins une des différences

$$
(1,4)=0, \quad(2,5)=0, \quad(3,6)=0 .
$$

On voit encore que le troisième invariant:

$$
I_{12}^{(\varrho)}=\Sigma[(1,2)(2,3)(3,1)]^{6} \times[(4,5)(4,6) \ldots(6,7)]^{4}
$$

reste différent de zéro pour la même supposition, parceque dans le terme sous le signe $\boldsymbol{\Sigma}$ il n'y a aucune de ces différences nulles.

Il est donc tout à fait impossible que $I_{4}^{(\rho)}$ soit un facteur de $I_{12}^{(\rho)}$. 
54 Schramm: Gritères pour les racines d'una équation.

Les formules générales développées en IV. nous donnent encore les critères pour la coëxistence de 4 racines imaginaires, deux à deux égales savoir pour

$$
I_{40}^{(\prime)}=0
$$

$$
\text { et } I_{4}^{(\varrho)}=\mathbf{\Sigma} A_{1}^{4} A_{2}=-\quad \text { ou aussi } \quad I_{12}^{\varrho}=\mathbf{\Sigma} A_{1}^{6} A_{2}^{4}=-
$$

car en désignant par $\mu$ l'exposant plus haut de la puissance imaginaire $(\sqrt{-1}) \mu$, on trouve pour le premier invariant: $\mu-1=10=4 \cdot 2+2$ et pour le second $\mu=14=4 \cdot 3+2$, pendant que les invariants:

$$
I_{20}^{(r, s)} \text { et } I_{12}^{(r, s)}
$$

donnent pour 4 racines imaginaires les valeurs respectives de $\mu-1$

$$
\mu-1=60=4 \cdot 15, \quad \mu-1=40=4 \cdot 10
$$

tant qu'ils ne seront plus applicables dans le cas mentionné.

Ces résultats suffiront peut-être à démontrer la possibilité d'une telle discussion des équations algébriques, d'un degré quelconque, au moyen des critères invariantifs. 